\title{
Nanomedicine: Application Areas and Development Prospects
}

\author{
Houria Boulaiz ${ }^{1}$ *, Pablo J. Alvarez ${ }^{1}$, Alberto Ramirez ${ }^{1}$, Juan A. Marchal ${ }^{1}$, Jose Prados ${ }^{1}$, \\ Fernando Rodríguez-Serrano ${ }^{1}$, Macarena Perán ${ }^{2}$, Consolación Melguizo ${ }^{1}$ and \\ Antonia Aranega ${ }^{1, *}$
}

1 Department of Human Anatomy and Embryology, Institute of Biopathology and Regenerative Medicine (IBIMER), School of Medicine, University of Granada, Granada 18071, Spain;

E-Mails: pablo.alvarez.exts@juntadeandalucia.es (P.J.A.); alrari80@ gmail.com (A.R.); jmarchal@ugr.es (J.A.M.); jcpradois@ugr.es (J.P.); fernrs@ugr.es (F.R.-S.); melguizo@ugr.es (C.M.) Department of Health Sciences, University of Jaén, Jáen 23071, Spain; E-Mail: mperan@ ujaen.es

* Authors to whom correspondence should be addressed; E-Mails: hboulaiz@ugr.es (H.B.); aranega@ugr.es (A.A.); Tel.:+34-958-243534; Fax: +34-958-246296.

Received: 31 March 2011; in revised form: 6 May 2011 / Accepted: 16 May 2011 /

Published: 19 May 2011

\begin{abstract}
Nanotechnology, along with related concepts such as nanomaterials, nanostructures and nanoparticles, has become a priority area for scientific research and technological development. Nanotechnology, i.e., the creation and utilization of materials and devices at nanometer scale, already has multiple applications in electronics and other fields. However, the greatest expectations are for its application in biotechnology and health, with the direct impact these could have on the quality of health in future societies. The emerging discipline of nanomedicine brings nanotechnology and medicine together in order to develop novel therapies and improve existing treatments. In nanomedicine, atoms and molecules are manipulated to produce nanostructures of the same size as biomolecules for interaction with human cells. This procedure offers a range of new solutions for diagnoses and "smart" treatments by stimulating the body's own repair mechanisms. It will enhance the early diagnosis and treatment of diseases such as cancer, diabetes, Alzheimer's, Parkinson's and cardiovascular diseases. Preventive medicine may then become a reality.
\end{abstract}

Keywords: nanomedicine; nanostructures; early diagnosis; drug delivery 


\section{Introduction}

The term nanotechnology refers to the ability to measure, design and manipulate materials at atomic, molecular and supramolecular level in order to understand, create and apply structures and systems with specific functions attributable to their size. Nanotechnology classically refers to matter in the size range of $1-100 \mathrm{~nm}$, but it is often extended to include materials below $1 \mu \mathrm{m}$ in size. A key goal is to assemble nanoparticles and integrate them into ordered structures in order to obtain useful materials. Although new, the advent of nanomaterials was forecast as long ago as 1959 by Richard P. Feynman [1]. Nanotechnology has been embraced by multiple industrial sectors for application in the field of electronic storage systems [2], biotechnology [3], magnetic separation and preconcentration of target analytes and targeted drug delivery [4,5], and as vehicles for gene and drug delivery [2,4,5]. Advances in nanotechnology have led to the development of new nanomaterials whose physiochemical properties differ from those of their larger counterparts due to their higher surface-to-volume ratio. These novel properties make them excellent candidates for biomedical applications, given the range of biological processes that occur at nanometer scale [6]. Nanotechnology is a new discipline of science and engineering that has led to innovative approaches in many areas of medicine. Its applications in the screening, diagnosis, and treatment of disease are collectively referred to as "nanomedicine" - an emerging field that has the potential to revolutionize individual and population-based health this century [7]. It is now possible to provide therapy at a molecular level with the help of nanoparticles, treating diseases and adding to our understanding of their pathogenesis. Nanomedicine can be considered a refinement of molecular medicine, integrating innovations in genomics and proteomics on the road to a more personalized medicine.

The impact of nanotechnology in medicine can mainly be seen in diagnostic methods, drug-release techniques and regenerative medicine.

Diagnostic methods are essential for the early detection of diseases to enable their prompt treatment, minimizing possible damage to the rest of the organism. The importance of imaging methods to diagnose, treat and follow up cancer, cardiovascular and neurological patients is well known. Diagnostic techniques based on the use of nanoparticles offer higher sensitivity and assist the early detection of disease, offering a better prognosis and greater possibilities of successful treatment [8]. Once the diagnosis is established, the fight against the disease begins, with medicaments playing a major role. There are numerous obstacles to the development of novel drugs against disease. Conventional drugs suffer from the major limitation of adverse effects, the result of the non-specificity of their action, and from a lack of effectiveness due to improper or ineffective dosages, e.g., in cancer chemotherapy and anti-diabetic therapy. Nanotechnology offers the possibility of designing novel drugs with greater cell specificity and new drug-release systems that act selectively on specific targets and protect the drug from degradation en route. This allows the administration of smaller but more effective doses, minimizing adverse effects. Nanotechnology can also be used to optimize drug formulations, increasing drug solubility and altering the pharmacokinetics to sustain the release of the drug, thereby prolonging its bioavailability. The diverse platforms of nanotechnology can be utilized to develop more sophisticated, cell-targeted therapies and to combine different drugs into a single nanotherapeutic agent for synergistic therapeutic benefits [9]. 
Regenerative medicine has been proposed as a highly promising approach to numerous degenerative diseases. To this end, nanomedicine employs gene therapy, cell therapy, tissue engineering, biomaterials and signaling molecules.

\section{Nanomedicine Applications}

Nanomedicine applications are grouped below in three interrelated areas: analytical/diagnostic tools, drug delivery and regenerative medicine (Figure 1).

Figure 1. Nanomedicine application areas.

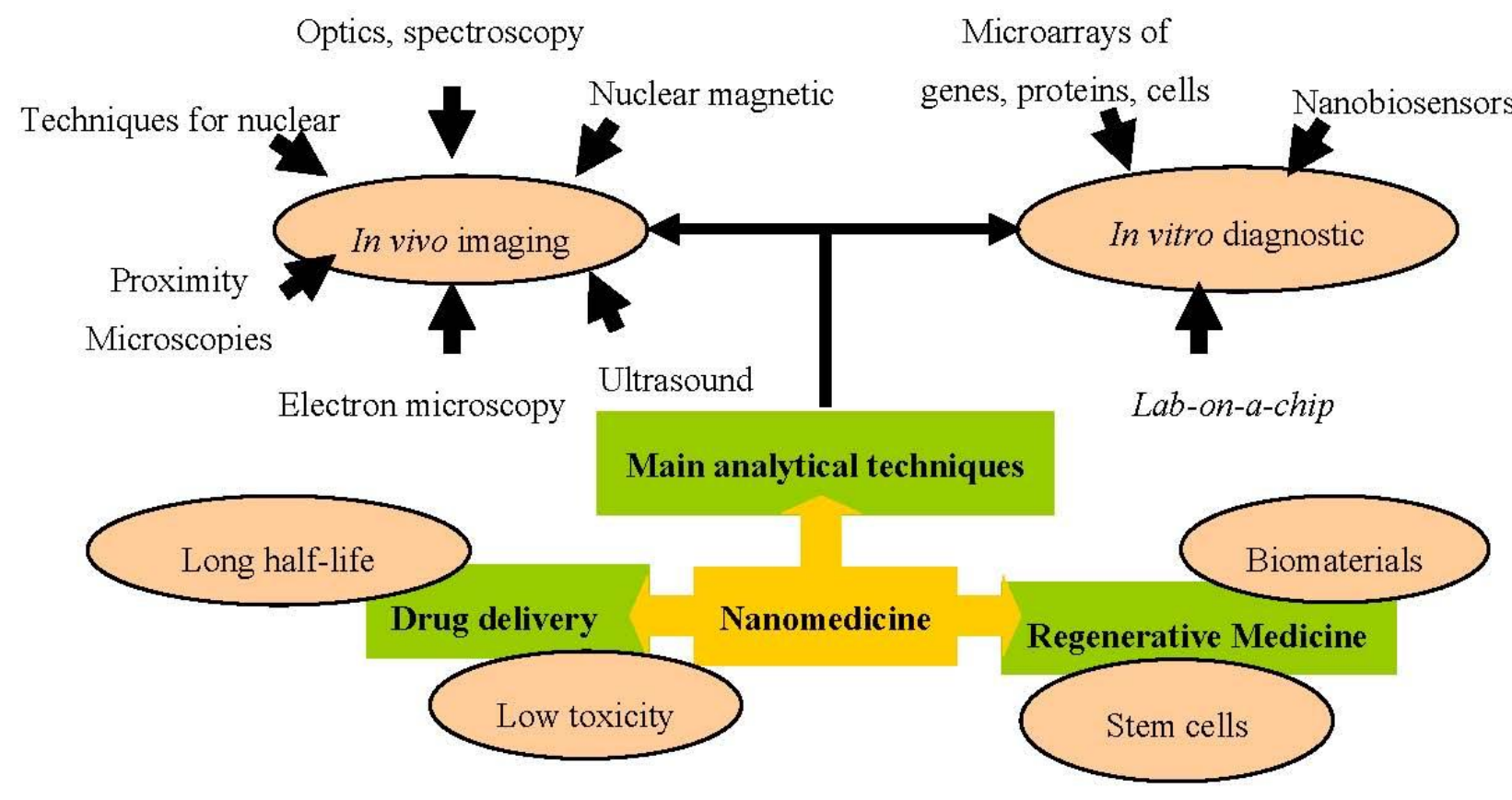

\subsection{Analytical and Diagnostic Tools}

The limitations of current diagnostic technology mean that some diseases can only be detected when at a very advanced stage. Nanodiagnostics, defined as the use of nanotechnology for clinical diagnostic purposes [10,11], was developed to meet the demand for increased sensitivity in clinical diagnoses and earlier disease detection.

The application of micro and nanobiotechnology in medical diagnostics can be subdivided into two broad categories: In vitro diagnostic devices and in vivo imaging. Research in this field is highly multi-disciplinary and there are close relationships among diagnostics, drug release and regenerative medicine, which are described in the following sections.

\subsubsection{In Vitro Diagnostic Devices}

The grounds for modern medicine were already laid in the middle of the 19th century with the recognition of the cell as the source of health and disease. Basic research to yield a better understanding of the highly complex working of cells became mandatory in medicine. The resulting improvements in methods to characterize cells or cell compartments in vitro (e.g., optical and 
luminescence microscopy, scanning probe microscopy, electron microscopy and imaging mass-spectrometry) have been critically important for the development of nanomedicine.

The miniaturization and integration of different functions in a single device, based on nanotechnology-derived techniques, have led to a new generation of devices that are smaller, faster and cheaper, require no special skills and give accurate readings. They require much smaller samples, implying less invasive and traumatic sample extraction methods, and deliver more complete and more accurate biological data from a single measurement. The use of these devices in research has become routine and has improved our understanding of the molecular basis of disease and helped to identify new therapeutic targets. In vitro diagnostic devices include nanobiosensors, microarrays, biochips of different elements (DNA, proteins or cells) and lab-on-a-chip devices.

\subsubsection{Nanobiosensor}

A nanobiosensor is defined as a compact analysis device that incorporates biological (nucleic acid, enzyme, antibody, receptor, tissue, cell) or biomimetic (macrophage-inflammatory proteins, aptamers, peptide nucleic acids) recognition elements [11,12]. Interaction between the compound or microorganism of interest and the recognition element produces a variation in one or more physical-chemical properties (e.g., pH, electron transfer, heat, potential, mass, optical properties, etc.) that are detected by the transducer. The resulting electronic signal indicates the presence of the analyte of interest and its concentration in the sample. These sensors can be electronically gated to respond to the binding of a single molecule. Prototype sensors have been successfully used to detect nucleic acids, proteins and ions. They can operate in liquid or gas phase, opening up an enormous variety of downstream applications. These detection systems use inexpensive low-voltage measurement methods and detect binding events directly, so there is no need for costly, complicated and time-consuming chemical labeling, e.g., with fluorescent dyes, or for bulky and expensive optical detection systems. As a result, these sensors are inexpensive to manufacture and portable [10]. Hence, nanobiosensors are revolutionizing the in vitro diagnosis of diseases and have major implications for human health. They allow healthcare professionals to simultaneously measure multiple clinical parameters using a simple, effective and accurate test. These devices are also ideal for high-throughput screening and for the detection of a single disease in various samples or of various diseases in a single sample [13].

\subsubsection{Microarrays}

The microarray is another diagnostic device that is becoming a standard technology in research laboratories worldwide. Since their first application, microarray technologies have proven productively functional in almost all areas of biomedical research [14-21]. The emergence of this new tool has allowed investigators to address previously intractable problems and identify novel potential therapeutic targets. They are using microarray technology to identify cardinal aspects of growth and development and explore the underlying genetic causes of numerous human diseases [14]. Microarray-based studies have enormous potential in the exploration of diseases such as cancer [15] and in the design and development of new drugs [16]. Microarrays have been widely applied in the study of various pathological conditions, including inflammation [17], atherosclerosis [18], breast 
cancer [19,20], colon cancer [21] and pulmonary fibrosis [22]. As a result, functions have been assigned to previously unannotated genes, and genes have been grouped into functional pathways [23,24].

Several types of microarray have been developed for different target materials, which can be DNA, cDNA, mRNA, protein, small molecules, tissues or any other material that can be quantitatively analyzed. DNA microarray technology has progressed rapidly over the past 10 years and allows the large-scale quantification of gene expression. A DNA array consists of a large number of DNA molecules orderly arranged on a solid substrate to form a matrix of sequences in two dimensions [25]. cDNA microarrays and oligonucleotide microarrays, called 'expression chips', are used for microarray expression analysis, i.e., to determine the level or volume of expression of a given gene. Single nucleotide polymorphism (SNP) microarrays detect mutations or polymorphisms in a gene sequence [26]. This technology is used to test an individual for disease expression patterns and to determine whether or not individuals are susceptible to a disease. Microarray comparative genomic hybridisation [27] is employed to seek genomic gains and losses or changes in the number of copies of a gene implicated in a disease. Protein microarrays are comprised of protein detectors (usually antibodies) arranged systematically over a glass slide and allow the investigation of expression profiles and the precise definition of protein functions in relation to biological processes [28]. Tissue microarrays represent one of the most valuable approaches in the microarray field, because they allow the simultaneous analysis of protein, RNA and DNA expression in multiple tissue samples [29].

The main applications of microarrays in human health are listed below [30].

- Gene expression analysis, used to determine gene expression patterns and simultaneously quantify the expression of a large number of genes, permitting comparison of their activation between healthy and diseased tissues.

- Detection of mutations and polymorphisms, allowing the study of all possible polymorphisms and the detection of mutations in complex genes.

- Sequentiation, used to sequence short DNA fragments (sequencing of long DNA fragments has not yet proven possible, although they can be used as quality controls).

- Therapy follow-up, allowing evaluation of genetic features that may affect the response to a given therapy.

- Preventive medicine, developing knowledge on the genetic features of diseases in order to treat and prevent them before symptom onset.

- Drug screening and toxicology, analyzing changes in gene expression during the administration of a drug, as well as localizing new possible therapeutic targets and testing for associated toxicological effects.

- Clinical diagnosis, allowing the rapid identification of pathogens by employing the appropriate genetic markers.

In conclusion, molecular diagnosis is a fast-growing field. Analysis of global expression by microarray techniques simultaneously reveals the state of thousands of genes of diseased cells. These approaches offer a more accurate diagnosis and risk assessment for various diseases, leading to a more precise prognosis and new therapeutic approaches. The ultimate reach of microarray technology will be achieved with its entry into the physician's clinic as a routine diagnostic tool [31]. 


\subsubsection{Lab-on-a-Chip}

The latest in vitro diagnostic development derives from the integration of several functions in a single device. Lab-on-a-chip integrates one or several laboratory functions on a single chip ranging from only a few millimeters to a square centimeter in size [32] and incorporates sample preparation, purification, storage, mixing, detection and other functions. Its development was based on advances in microsystem technologies and in the field of microfluidics on the design of devices that use microscopic volumes of sample. The chips use a combination of phenomena, including pressure, electroosmosis, electrophoresis and other mechanisms to move samples and reagents through microscopic channels and capillaries, some as small as a few dozen nanometers.

Lab-on-a-chip has many applications in medicine and biology. These devices are likely to have a significant socio-economic impact, bringing sophisticated analytical tools to Third World countries, rural areas and resource-poor regions. Advantages of their use include the extremely rapid analysis of samples containing fluid volumes that can be less than a picoliter, the high degree of automation, cost savings due to the low consumption of reagents and samples and their portable and disposable nature. Lab-on-a-chip is used in real-time polymerase chain reaction [33] and immunoassays [34] to detect bacteria, viruses and cancers. It can also be used in blood sample preparation to crack cells and extract their DNA [32]. Lab-on-a-chip may soon play an important role in efforts to improve global health, especially with the development of point-of-care testing devices. Infectious diseases that are treatable in developed nations are often deadly in countries with limited healthcare resources. In some cases, healthcare clinics possess a drug to treat a given disease but lack the diagnostic tool to identify the patients who should receive it. Many researchers believe that lab-on-a-chip technology will be the key to powerful new diagnostic instruments. The goal is to create microfluidic chips that will allow healthcare providers in poorly-equipped clinics to perform diagnostic tests (e.g., immunoassays and nucleic acid assays) with no laboratory support. One active research line on the lab-on-a-chip addresses the diagnosis and management of HIV infections [35]. Around 40 million people are infected with HIV in the world, yet only 1.3 million receive antiretroviral treatment and around $90 \%$ of HIV-infected individuals have never been tested for the disease. This is largely because its diagnosis requires measurement of the number of $\mathrm{CD} 4+\mathrm{T}$ lymphocytes in the blood by means of flow cytometry, a complicated technique that requires trained technicians and expensive equipment that are not available in most developing regions.

\subsubsection{In Vivo Imaging}

Imaging systems can be grouped according to the energy used to derive the visual information (X-rays, positrons, photons or sound waves), the spatial resolution (macroscopic, mesoscopic or microscopic) or the type of information obtained (anatomical, physiological, cellular or molecular). Unlike classic imaging diagnosis with computed tomography (CT), magnetic resonance imaging (MRI) or ultrasounds, nano-imaging or molecular imaging includes techniques designed to obtain molecular data to identify the causes of the disease in vivo rather than its eventual consequences. Nanotechnology has produced advances in imaging diagnosis, developing novel methods and increasing the resolution and sensitivity of existing techniques. Although these systems have emerging recently and 
only some of them are in clinical and preclinical use, they have made it possible to study human biochemical processes in different organs in vivo, opening up new horizons in instrumental diagnostic medicine. These systems include positron-emission tomography (PET), single-photon-emission CT (SPECT), fluorescence reflectance imaging, fluorescence-mediated tomography (FMT), fiber-optic microscopy, optical frequency-domain imaging, bioluminescence imaging, laser-scanning confocal microscopy and multiphoton microscopy [36].

Imaging diagnosis has gained importance over the years and is now an indispensable diagnostic tool for numerous diseases, including cancer, cardiovascular diseases and neurological syndromes. The main benefits of molecular imaging for in vivo diagnosis lie in the early detection of disease and the monitoring of disease stages, e.g., in cancer metastasis [37], supporting the development of individualized medicine and the real-time assessment of therapeutic and surgical efficacy. An ideal imaging modality should be non-invasive, sensitive, and provide objective information on cell survival, function and localization. MRI, CT, PET) and SPECT are the most widely used and studied modalities in cancer patients [36,38,39] Overall, nuclear imaging by PET or SPECT offers greater sensitivity ( $>5 \times 10^{3}$ cells) [40] but is limited by the lack of anatomical context [41], whereas MRI provides accurate anatomical detail but no data on cell viability and shows poor sensitivity ( $>10^{5}$ cells) [42]. Although none of these modalities is ideal, MRI is the preferred option for cellular tracking $[39,43,44]$ By detecting proton relaxations in the presence of a magnetic field (1.5 Tesla [T]-3 $\mathrm{T}$ for clinical imaging), it yields tomographic images with excellent soft tissue contrast and can locate the cells of interest in the context of the surrounding milieu (oedema or inflammation) $[45,46]$ without the use of harmful ionizing radiations (the case with CT, PET or SPECT). In addition, MRI offers a longer tracking window in comparison to PET and SPECT, which are limited by the decay of the short-lived radioactive isotopes.

In parallel to the development of imaging techniques, intense research has been fuelled by the need for practical, robust and highly sensitive and selective detection agents that can address the deficiencies of conventional technologies. New contrast agents, used to increase the sensitivity and contrast of imaging techniques are increasingly complex and formed by synthetic and biological nanoparticles. Nanoparticles possess certain size-dependent properties, particularly with respect to optical and magnetic parameters, which can be manipulated to achieve a detectable signal [47]. The primary event in most nanoparticle-based assays is the binding of a nanoparticle label or probe to the target biomolecule that will produce a measurable signal characteristic of the target biomolecule. A probe that is to function in a biological system must be water-soluble and stable and have minimal interaction with the surrounding environment. For fluorescence readouts, the probe should ideally have a high fluorescence quantum yield and minimal photobleaching rates in order to generate a detectable signal [48]. The most promising nanotechnologies for clinical diagnosis include quantum dots (QDs), gold nanoparticles and cantilevers, whose main features are summarized in Table 1. 
Table 1. Comparison of quantum dots (QDs), cantilevers and gold nanoparticles.

\begin{tabular}{|c|c|c|c|}
\hline Feature & QDs & Cantilevers & Gold nanoparticles \\
\hline Structure & $\begin{array}{l}\text { Semiconductor nanocrystals } \\
\text { typically composed of a } \\
\text { semiconductor core encapsulated } \\
\text { by another semiconductor shell } \\
\text { with a larger spectral band-gap; a } \\
\text { third silica shell can be added for } \\
\text { water solubility }\end{array}$ & $\begin{array}{l}\text { Nano-machined silicon or a } \\
\text { piezoelectric } \\
\text { material such as quartz similar } \\
\text { to those used in atomic force } \\
\text { microscopy }\end{array}$ & $\begin{array}{l}\text { Gold particles in the nanometre } \\
\text { size domain; gold nanoshells } \\
\text { consist of concentric sphere } \\
\text { nanoparticles with a dielectric } \\
\text { core (typically gold sulfide or } \\
\text { silica) surrounded by a thin gold } \\
\text { shell }\end{array}$ \\
\hline Size & $2-10 \mathrm{~nm}$ & Nanoscale & $\begin{array}{l}2-150 \mathrm{~nm} \\
\text { (changes in optical properties as } \\
\text { a function of size) }\end{array}$ \\
\hline $\begin{array}{l}\text { Diagnostic } \\
\text { applications }\end{array}$ & $\begin{array}{l}\text { - In vitro diagnosis: } \\
\text { immunohistochemistry, infectious } \\
\text { agent detection, } \\
\text { fluoroimmunoassays, } \\
\text { immunoassays, intracellular } \\
\text { imaging and tissue imaging. } \\
\text { - In vivo imaging }\end{array}$ & $\begin{array}{l}\text { DNA and protein (various } \\
\text { biomarkers) detection and } \\
\text { quantification. }\end{array}$ & $\begin{array}{l}\text { Detection of DNA and proteins } \\
\text { (including antibodies) }\end{array}$ \\
\hline $\begin{array}{l}\text { Method for } \\
\text { detecting }\end{array}$ & $\begin{array}{l}\text { Fluorometry and several types of } \\
\text { microscopy, such as fluorescence, } \\
\text { confocal, total internal reflection, } \\
\text { wide-field epifluorescence, atomic } \\
\text { force, and multiphoton } \\
\text { microscopy }\end{array}$ & $\begin{array}{l}\text { Operate either statically, by } \\
\text { measuring absolute cantilever } \\
\text { deflection, or dynamically, by } \\
\text { measuring resonance } \\
\text { frequency shifts }\end{array}$ & $\begin{array}{l}\text { Surface plasmon resonance } \\
\text { microscopy. Gold particles } \\
\text { coated }^{\text {with }} \text { silver have strong } \\
\text { light-scattering properties and } \\
\text { can easily be detected by } \\
\text { standard dark-field microscopy } \\
\text { with white light illumination }\end{array}$ \\
\hline Advantage & $\begin{array}{l}\text { - Their optical tunability, } \\
\text { resistance to photobleaching, } \\
\text { excitation of various QDs by a } \\
\text { single wavelength of light (for } \\
\text { multiplexing), narrow emission } \\
\text { band, and exceptional stability of } \\
\text { optical properties after } \\
\text { conjugation to a biomolecule. } \\
\text { - They do not need lasers for } \\
\text { excitation. } \\
\text { - The instrumentation needed for } \\
\text { detection is simple. }\end{array}$ & $\begin{array}{l}\text { - Their sensitivity, } \\
\text { compatibility with silicon } \\
\text { technology, and capacity for } \\
\text { microfluidic integration. } \\
\text { - Good potential for high } \\
\text { throughput protein screening }\end{array}$ & $\begin{array}{l}\text { Their optical properties, useful } \\
\text { for imaging and photothermal } \\
\text { therapy. } \\
\text { Their surfaces, functionalized } \\
\text { using various well-characterized } \\
\text { chemical moieties (thiols, } \\
\text { disulfides, amines) }\end{array}$ \\
\hline Toxicity & $\begin{array}{l}\text { Risk of leakage of toxic core } \\
\text { semiconductor materials into host } \\
\text { system or into the environment on } \\
\text { disposal }\end{array}$ & $\begin{array}{l}\text { No particular toxicity } \\
\text { concerns }\end{array}$ & No particular toxicity concerns \\
\hline
\end{tabular}




\subsection{Drug Delivery}

One of the most important nanotechnology applications developed over the past decade have been nanovehicles, nanoscale compounds used as a therapeutic tool and designed to specifically accumulate in the sites of the body where they are needed in order to improve pharmacotherapeutic outcomes. The main objective of this application is to increase therapeutic effectiveness while obtaining lower toxicity rates. Hence, nanodrugs and nanodiagnostics have been developed to increase bioavailability profiles, enabling the administration of lower doses and thereby minimizing the adverse reactions found with conventional drugs in clinical practices and increasing the quality of patient health [49]. Table 2 compiles some drugs using nanocarriers and their route of administration.

Table 2. Some drugs using nanocarriers and their administration routes.

\begin{tabular}{|c|c|c|}
\hline Compounds & Nanocarrier & Application \\
\hline CPX-1 irinotecan & Liposome & Systemic \\
\hline DNA (gene therapy) & Solid lipid nanoparticles & Systemic \\
\hline Cancer vaccine & $\begin{array}{l}\text { Immunostimulatory acid-degradable } \\
\text { microparticles }\end{array}$ & Subcutaneously \\
\hline Camptothecin & Polymeric nanoparticles & Systemic \\
\hline Tamoxifen citrate & Solid lipid nanoparticles & Systemic \\
\hline Pilocarpine hydrochloride & Polymeric nanoparticles & Systemic \\
\hline Clotrimazole & $\begin{array}{l}\text { Solid lipid nanoparticles and nanostructured } \\
\text { lipid carriers }\end{array}$ & Topical \\
\hline Clozapine & Solid lipid nanoparticles & Systemic \\
\hline Coenzyme Q 10 & Solid lipid nanoparticles & Topical \\
\hline Titanium dioxide & Solid lipid nanoparticles & Topical \\
\hline 5-Fluorouracil & Nanostructured lipid carriers & Systemic \\
\hline Ibuprofen & Solid lipid nanoparticles & Topical \\
\hline Insulin & Solid lipid nanoparticles & Systemic \\
\hline Isotretinoin & Solid lipid nanoparticles & Systemic \\
\hline Ketoconazole & Solid lipid nanoparticles & Topical \\
\hline Mifepristone & Solid lipid nanoparticles & Systemic \\
\hline $\begin{array}{l}N, N \text {-Diethyltoluamide } \\
\text { (DEET) }\end{array}$ & Solid lipid nanoparticles & Topical \\
\hline$N$-dodecyl-ferulate & Solid lipid nanoparticles & Systemic \\
\hline Oxybenzone & Solid lipid nanoparticles & Topical \\
\hline Clobetasol propionate & Nanostructured lipid carriers & Systemic \\
\hline Retinoids & Solid lipid nanoparticles & Topical \\
\hline Triptolide & Solid lipid nanoparticles & Systemic \\
\hline Vitamin A & Solid lipid nanoparticles & Topical \\
\hline MCC465 doxorubicin & mAb-liposome & Systemic \\
\hline NC-6004 cisplatin & Micelle & Systemic \\
\hline NK105 paclitaxel & Micelle & Systemic \\
\hline NK911 doxorubicin & Micelle & Systemic \\
\hline PK1 doxorubicin & HPMA copolymer & \\
\hline SP1049C doxorubicin & Micelle & Systemic \\
\hline
\end{tabular}


Table 2. Cont.

\begin{tabular}{|c|c|c|}
\hline Compounds & Nanocarrier & Application \\
\hline Etoposide & Nanostructured lipid carriers & Systemic \\
\hline Docetaxel & Nanostructured lipid carriers & Systemic \\
\hline Paclitaxel & Nanostructured lipid carriers \\
\hline Paclitaxel & Polymeric nanoparticles & Subcutaneously \\
\hline
\end{tabular}

In the field of cancer therapy there are a lot of clinical applications based on nanotechnologies, with a major development in drug delivery systems. The reason for the rise in nanotechnology applications in medicine is the prospect of improving effectiveness by the biological targeting of drugs in current clinical use [50]. In cancer treatments, nanoparticles are usually administered by intravenous injection, travelling in the blood stream and passing through biological barriers (cell membranes) of the organism in order to reach and activate their molecular targets [51]. One of the main objectives of nanotechnology is overcome the shortcomings of classical chemotherapy, including the multiple drug resistance mechanisms that make this treatment ineffective in a high percentage of cancer cases. The other problem of conventional anticancer therapies is the non-specific action of the drugs, leading them to damage both tumor and non-tumor cells in a state of division.

Nanoparticles can overcome the side effects of conventional therapies by the following means: (1) sustaining drug release over time; (2) so-called "passive enhanced permeability", targeting the effect to tumor tissue; (3) targeting the cell surface with the use of ligands related to endosomal uptake and membrane disruption; (4) permitting release of the drug in the cell cytoplasm; and (5) protecting the drug from enzymatic degradation [52,53]. The main goals of drug delivery design are: (i) to decrease the side effects of conventional therapy by decreasing drug concentration in normal tissues; (ii) to enhance the pharmacokinetics and pharmacodynamics profiles; (iii) to allow intravenous drug administration by increasing drug solubility; (iv) to minimise drug loss in transit and maximize drug concentration in the tumor; (v) to improve drug stability by avoiding drug degradation; (vi) to achieve optimal cellular uptake and intracellular delivery; and (vii) to ensure biocompatibility and biodegradability [54].

Alternative methods have been developed to increase the effectiveness of drug delivery using the passive or active targeting capacities of nanovehicles. The passive targeting form of drug delivery consists of transporting nanoparticles through leaky tumor capillary fenestrations within the tumor mass to reach cells by convection or passive diffusion. Convection is related to the movement of molecules through organic fluids and is the predominant transport mode for a high percentage of molecules across large pores. However, compounds with a low molecular weight (e.g., oxygen) are most frequently transported by diffusion, in which molecules cross the cell membrane along a concentration gradient, with no energy cost to the cell. In fact, convection through the tumor mass is poor, due to interstitial hypertension; therefore, diffusion is the main type of drug transport used, accumulating nanoparticles with drug by the so-called Enhanced Permeability and Retention (EPR) effect. Nanoparticles are able to achieve an optimal EPR effect if they can evade the immune response, and they remain highly stable in the blood stream until reaching the tumor. The EPR effect is a physiological process based on the capacity of long-circulating nanoparticles to penetrate through the 
leaky tumor-formed vasculature and degrade within the tumor, releasing the drug and achieving a local concentration in solid tumors that is several-fold higher than that obtained with free drugs [55].

Very high local concentrations of drug-loaded nanoparticles have been obtained within tumors, from 10- to 50-fold higher in comparison to normal tissues in a time period of 1-2 days. The achievement of these drug concentrations in the tumor requires nanoparticles to possess the following characteristics: (a) nanoparticle size between 10 and $100 \mathrm{~nm}$; (b) a neutral or anionic nanoparticle surface charge to prevent elimination by the kidneys; and (c) the ability to avoid opsonization and phagocytosis, which destroy foreign material via the reticuloendothelial system [54,56].

Active targeting using nanoparticles as the delivery system allows a specific area of the body to be targeted, avoiding one of the drawbacks of current chemotherapy, i.e., toxic effects in non-malignant organs [57]. Studies are being carried out on the attachment of targeting ligands on the nanoparticle surface, enabling specific binding of the nanoparticle to receptors on the tumor cell surface. Ligands that bind to specific overexpressed receptors are selected. The types of ligand used include antibodies, antibody fragments or non-antibody (peptidic) ligands. Over the past few years, considerable interest has developed in the numerous antigens present on tumor cells. For instance, HER2 receptor has been proposed as a therapeutic target. HER2 gene, also known as c-erbB2 and neu, encodes a 185-kDa transmembrane glycoprotein receptor that belongs to the ErbB family of growth factor receptors with intrinsic tyrosine kinase activity, whose members exist in homodimer and heterodimer form when activated [58]. Trastuzumab is a humanized antibody designed to specifically recognize the HER-2 receptor and was approved by the FDA in 1998 for the treatment of metastatic breast cancer. Different studies have shown that normal cells can express moderate amounts of HER2 target antigen, while it is overexpressed in tumors in certain patients, and this difference between healthy tissues and tumors allows the effective use of this antibody in patients [59].

Fibroblast growth factor receptors (FGFRs) have also become potential targets for drug delivery and cancer therapy. Fibroblast growth factors (FGFs) are small peptide growth factors that play an important role in tumor growth and angiogenesis because of their high affinity to heparin. FGFRs are upregulated versus normal tissues in numerous tumor cells and tumor neovasculature in situ [60]. One candidate as a targeting ligand for tumor cells is the peptide KRTGQYKLC (bFGFp), which could interact with FGFR1 by binding to bFGF. The epidermal growth factor receptor (EGFR) is a receptor tyrosine kinase over-expressed on many human cancer cells surface, making it a target for anticancer drug delivery. Liposomes targeting this receptor promoted the effective intracellular delivery of doxorubicin to tumor cells, yielding superior anti-tumor effects in different in vivo assays. In previous studies, the peptide GE11 was identified as a novel ligand with high affinity towards EGFR and proved effective to mediate targeted liposome delivery to EGFR-positive tumors in vivo [61]. Investigations are ongoing into drug delivery systems based on nanoparticles with different geometry, compositions and surface modifications (Figure 2). They are providing researchers with an enormous collection of nanoparticles with promising applications [62]. 
Figure 2. Some drug delivery systems.

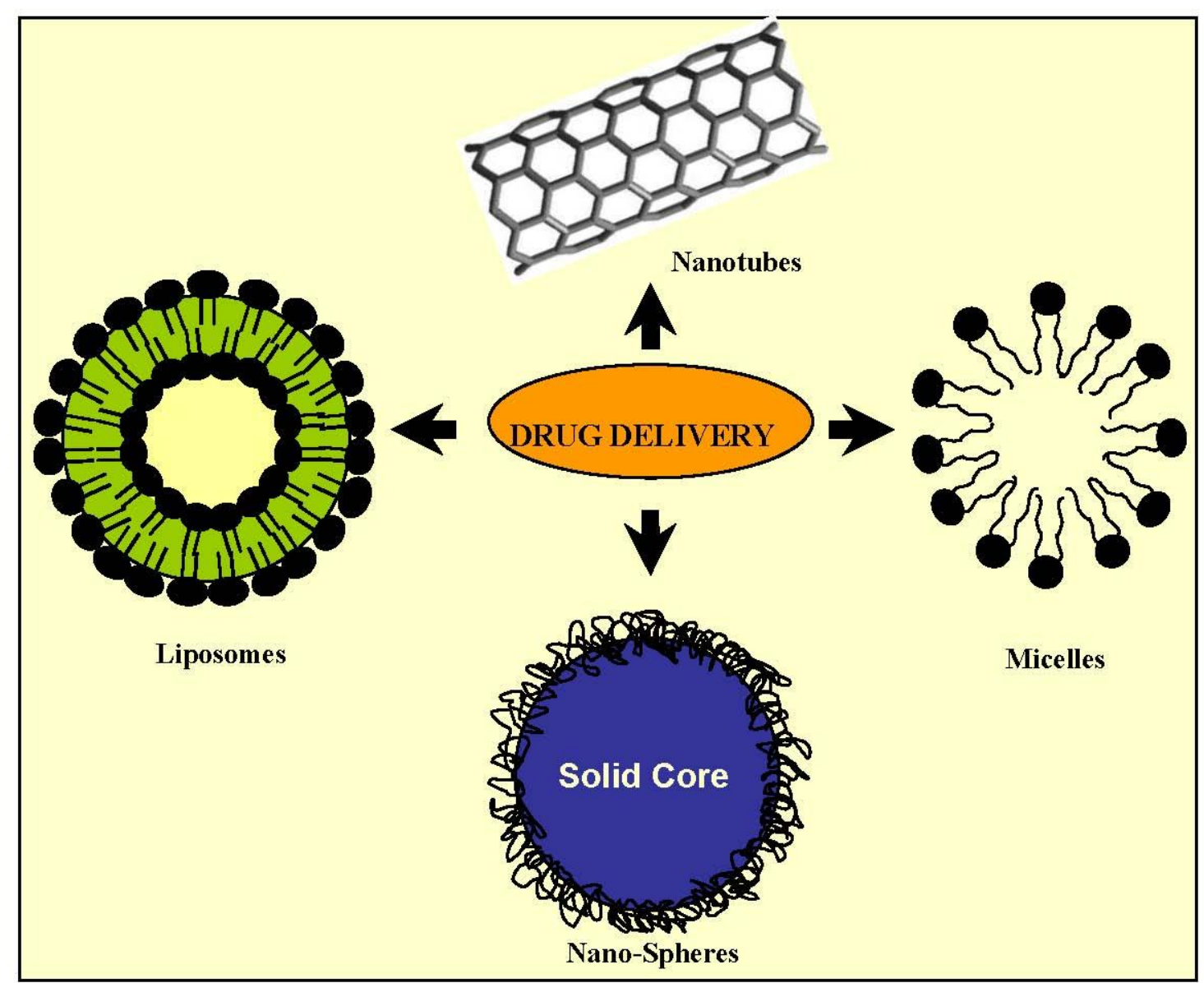

\subsubsection{Micelles}

A micelle is an aggregate of amphiphilic surfactant molecules spontaneously created on immersion in water, usually forming spherical vesicles. They contain a hydrophobic core in which hydrophobic substances, such as pharmacological compounds, can be introduced for release in different parts of the organism. A micelle typically comprises a hydrophilic or polar charged "head" group and a hydrophobic "tail", normally composed of a hydrocarbonated portion of long fatty acids. The characteristics of the surfactants that make up the micelle determine its size [63].

\subsubsection{Nanoemulsions}

Nanosized emulsions are composed of a mixture of two-phase insoluble liquids, in which vesicles in the dispersed phase are surrounded by the continuous phase. Different types of surfactants are used to stabilize the emulsion, preventing the dispersed phase from coalescing into a macroscopic phase [64]. Many emulsions used in drug delivery systems can be formulated on a nanosize scale. In these cases, the aqueous phase conform the continuous phase, and the drug is often carried in (or is itself) the non-aqueous liquid phase of the emulsion. Generally, the surfactant molecules used to stabilize micelles are the same as those forming the structural part of the nanoemulsions [65]. 


\subsubsection{Solid Nanoparticles}

This structure presents different characteristic features such as: (a) the material present in the centre of the particle forms a solid core at least at room temperature; (b) Solid nanoparticles not necessarily present in a spherical form. The geometry may change, presenting angular forms. This characteristic is particularly visible if the nanoparticle consists of crystals of a protein or another therapeutic agent. To avoid flocculation a surfactant is usually required to stabilize the nanoparticle [65].

\subsubsection{Dendrimers}

These are usually regularly-branched three-dimensional structures with a treelike form and a molecule as the central core. Branching units emerge from the central molecule by polymerization or are synthesized from the periphery and terminate at the central molecule. Branch lengths have steric limitations and dendrimer forms are sphere-shaped, with low molecular size but high molecular weight. These structures are used to transport drugs in two ways: (a) by attaching drug molecules to functional groups on the dendrimer surface; or (b) by enclosing the drugs in the dendritic channels within the sphere. The well-defined structure, size monodispersity, surface functionalization capability and stability of these nanoparticles make them a promising vehicle system for various agents (e.g., genes and anticancer drugs) by complexation or encapsulation [66,67]. One example is the formation of dendrimers with polymers such as polyamidoamine (PAMAM), which confer stability, availability and tolerability. The drug is contained in a central cavity and can be entrapped in channels between dendrons [23].

\subsubsection{Liposomes}

The effectiveness of liposomes as drug vehicles is related to their pharmokinetics and depends on the physicochemical conditions, e.g., size, surface charge, membrane lipid packing, steric stabilization, dose, and administration route [68]. A major advantage of liposomes is their long persistence in the blood, favoring the delivery of their contents to target tissues. Liposomes with diameters of 100-300 $\mu \mathrm{m}$ accumulate around tumors and are not subject to rapid clearance by the reticuloendothelial system [69]. Liposomes are vesicular nanostructures formed by a lipid bilayer composed of phospholipid and cholesterol molecules (structural components of cell membranes) characterized by extended, two-dimensional and clearly separated hydrophilic and hydrophobic regions. The hydrophilic portions of bilayer lipids are directed towards aqueous phases (external and internal), whereas the hydrophobic portions of both lipid layers are directed towards one another, forming an internal hydrophilic compartment that can encapsulate water-soluble drugs [70]. Actually there is a type of liposome well studied in clinical, the pegylated liposomes, which forms a water shell on the liposomal surface and provides a steric barrier to the liposomes for avoiding interactions with plasma proteins, resulting in escape from trapping by the reticuloendothelial system [71]. One example is liposomal-encapsulated doxorubicin, which has an elimination half-life of $55 \mathrm{~h}$ in comparison to only $0.2 \mathrm{~h}$ for doxorubicin in free form. The drugs are solubilized and protected from enzymatic degradation and inactivation either by physical entrapment within the nanoparticle or by conjugation 
with constituent components. Encapsulation into the liposomal carrier also causes a significant reduction in the most significant adverse side effect of doxorubicin, such as cardiotoxicity [72].

\subsection{Regenerative Medicine}

Tissue engineering brings together principles and innovations from engineering and the life sciences for the improvement, repair or replacement of tissue/organ function. Since its inception, this multidisciplinary field has been governed by the generic concept of combining cell, scaffold (artificial extracellular matrix) and bioreactor technologies in the design and fabrication of neo-tissues/organs [73]. Every tissue or organ in our body is composed of parenchymal cells (functional cells) and mesenchymal cells (support cells) contained within an extracellular matrix to form a microenvironment, and these microenvironments collectively form our tissues and organs. In terms of the development and maintenance of tissues and organs, our body is the "bioreactor", exposing the microenvironment of the cell and extracellular matrix to biomechanical forces and biochemical signals. The ultimate goal is to enable the body (cellular components) to heal itself by introducing a tissue engineered scaffold that the body recognizes as "self" and uses to regenerate "neo-native" functional tissues [74]. Furthermore, the demand for organs for transplantation far exceeds the supply, and the construction of organs by regenerative therapy has been presented as a promising option to address this deficit. Nanotechnology has the potential to provide instruments that can accelerate progress in the engineering of organs [75]. Achievement of the more ambitious goals of regenerative medicine requires control over the underlying nanostructures of the cell and extracellular matrix. Cells, typically microns in diameter, are composed of numerous nanosized components that all work together to create a highly organized, self-regulating machine. Cell-based therapies, especially those based on stem cells, have generated considerable excitement in the media and scientific communities and are among the most promising and active areas of research in regenerative medicine [76]. The pace of research could be accelerated by the creation of multi-functional tools to improve the monitoring and modification of cell behavior. While nanomedicine is primarily focused on cancer-related research, the application of nanotechnology has considerable potential in cell-based therapies for regenerative medicine, e.g., in localizing, recruiting and labeling stem cells to begin the regeneration process [75].

\section{Conclusions}

Nanotechnology is an emerging interdisciplinary field that combines biology, chemistry and engineering. It is expected to lead to major advances towards individualized medicine, improving the sensitivity and specificity of existing techniques to discover and detect biomarkers and developing novel nanodiagnostic instruments. This would allow earlier and more personalized diagnosis and therapy, improving the effectiveness of drug treatments and reducing side effects. In addition, nanoparticles are a promising platform technology for the synthesis of molecular-specific contrast agents. 


\section{Acknowledgements}

The study was supported by the Science and Innovation Ministry (Project no. SAF2009-12295). The authors are grateful to "Younes Taferssiti Zarouila" for the excellent linguistic assistance.

\section{References}

1. Appenzeller, T. The man who dared to think small. Science 1991, 254, 1300-1301.

2. Kang, Y.S.; Risbud, S.; Rabolt, J.F.; Stroeve, P. Synthesis and characterization of nanometer-size $\mathrm{Fe}_{3} \mathrm{O}_{4}$ and $\mathrm{g}-\mathrm{Fe}_{2} \mathrm{O}_{3}$ particles. Chem. Mater. 1996, 8, 2209-2211.

3. Pankhurst, Q.A.; Connoly, J.; Jones, S.K. Applications of magnetic nanoparticles in biomedicine. J. Phys. D. Appl. Phys. 2003, 36, 167.

4. Dobson, J. Gene therapy progress and prospects: magnetic nanoparticle-based gene delivery. Gene Ther. 2006, 13, 283-287.

5. Rudge, S.; Peterson, C.; Vessely, C.; Koda, J.; Stevens, S.; Catterall, L. Adsorption and desorption of chemotherapeutic drugs from a magnetically targeted carrier (MTC). J. Control. Release 2001, 74, 335-340.

6. Mody, V.V.; Siwale, R.; Singh, A.; Mody, H.R. Introduction to metallic nanoparticles. J. Pharm. Bioallied. Sci. 2010, 2, 282-289.

7. Zhang, L.; Gu, F.X.; Chan, J.M.; Wang, A.Z.; Langer, R.S.; Farokhzad, O.C. Nanoparticles in medicine: Therapeutic applications and developments. Clin. Pharmacol. Ther. 2008, 83, 761-769.

8. Surendiran, A.; Sandhiya, S.; Pradhan, S.C.; Adithan, C. Novel applications of nanotechnology in medicine. Indian J. Med. Res. 2009, 130, 689-701.

9. Zarbin, M.A.; Montemagno, C.; Leary, J.F.; Ritch, R. Nanotechnology in ophthalmology. Can. J. Ophthalmol. 2010, 45, 457-476.

10. Jain, K.K. Nanotechnology in clinical laboratory diagnostics. Clin. Chim. Acta 2005, 358, 37-54.

11. Jain, K.K. Nanodiagnostics: Application of nanotechnology in molecular diagnostics. Expert Rev. Mol. Diagn. 2003, 3, 153-161.

12. Singh, R.P.; Oh, B.K.; Ch, J.W. Application of peptide nucleic acid towards development of nanobiosensor arrays. Bioelectrochemisty 2010, 79, 153-161.

13. European Technology Platform on Nanomedicine. Nanotechnology for Health. Vision Paper and Basis for a Strategic Research Agenda for Nanomedicine. Eur. Comm. 2005, 1, 1-39.

14. Debouck, C.; Goodfellow, P.N. DNA microarrays in drug discovery and development. Nat. Genet. 1999, 21, 48-50.

15. Cole, K.A.; Krizman, D.B.; Emmert-Buck, M.R. Chuaqui, R.F.; Bonner, R.F.; Best, C.J. The genetics of cancer: A 3D model. Nat. Genet.1999, 21, 38-41.

16. Gerhold, D.L.; Jensen, R.V.; Gullans, S.R. Better therapeutics through microarrays. Nat. Genet. 2002, 32, 547-551.

17. Heller, R.A.; Schena, M.; Chai, A.; Shalon, D.; Bedilion, T.; Gilmore, J.; Woolley, D.E.; Davis, R.W. Discovery and analysis of inflammatory disease-related genes using cDNA microarrays. Proc. Natl. Acad. Sci. USA 1997, 94, 2150-2155. 
18. McCaffrey, T.A.; Fu, C.; Du, B.; Eksinar, S.; Kent, K.C.; Bush, H., Jr.; Kreiger, K.; Rosengart, T.; Cybulsky, M.I.; Silverman, E.S.; Collins, T. High-level expression of Egr-1 and Egr-1-inducible genes in mouse and human atherosclerosis. J. Clin. Invest. 2000, 105, 653-662.

19. Perou, C.M.; Jeffrey, S.S.; Van de Rijn, M.; Rees, C.A.; Eisen, M.B.; Ross, D.T; Pergamenschikov, A.; Williams, C.F.; Zhu, S.X.; Lee, J.C.; Lashkari, D.; Shalon, D.; Brown, P.O.; Botstein, D. Distinctive gene expression patterns in human mammary epithelial cells and breast cancers. Proc. Natl. Acad. Sci. USA 1999, 96, 9212-9217.

20. Perou, C.M.; Sørlie, T.; Eisen, M.B.; Rijn, M.; Jeffrey, S.S.; Rees, C.A.; Pollack, J.R.; Ross, D.T.; Johnsen, H.; Akslen, L.A.; Fluge, O.; Pergamenschikov, A.; Williams, C.; Zhu, S.X.; Lønning, P.E.; Børresen-Dale, A.L.; Brown, P.O.; Botstein, D. Molecular portraits of breast tumours. Nature 2000, 406, 747-752.

21. Alon, U.; Barkai, N.; Notterman, D.A.; Gish, K.; Ybarra, S.; Mack, D.; Levine, A.J. Broad patterns of gene expression revealed by clustering analysis of tumor and normal colon tissues probed by oligonucleotide arrays. Proc. Natl. Acad. Sci. USA 1999, 96, 6745-6750.

22. Kaminski, N.; Allard, J.D.; Pittet, J.F.; Zuo, F.; Griffiths, M.J.; Morris, D.; Huang, X.; Sheppard, D.; Heller, R.A. Global analysis of gene expression in pulmonary fibrosis reveals distinct programs regulating lung inflammation and fibrosis. Proc. Natl. Acad. Sci. USA 2000, 97, 1778-1783.

23. Stoughton, R.B. Applications of DNA microarrays in biology. Annu. Rev. Biochem. 2005, 74, 53-82.

24. Plomin, R.; Schalkwyk, L.C. Microarrays. Dev. Sci. 2007, 10, 19-23.

25. Schulze, A.; Downward, J. Navigating gene expression using microarrays - a technology review. Nat. Cell Biol. 2001, 3, 190-195.

26. Kennedy, G.C.; Matsuzaki, H.; Dong, S.; Liu, W.M.; Huang, J.; Liu, G.; Su, X.; Cao, M.; Chen, W.; Zhang, J.; Liu, W.; Yang, G.; Di, X.; Ryder, T.; He, Z.; Surti, U.; Phillips, M.S.; Boyce-Jacino, M.T.; Fodor, S.P.; Jones, K.W. Large-scale genotyping of complex DNA. Nat. Biotechnol. 2003, 21, 1233-1237.

27. Ulger, C.; Toruner, G.A.; Alkan, M.; Mohammed, M.; Damani, S.; Kang, J.; Galante, A.; Aviv, H.; Soteropoulos, P.; Tolias, P.P.; Schwalb, M.N.; Dermody, J.J. Comprehensive genome-wide comparison of DNA and RNA level scan using microarray technology for identification of candidate cancer-related genes in the HL-60 cell line. Cancer Genet. Cytogenet. 2003, 147, 28-35.

28. MacBeath, G. Protein microarrays and proteomics. Nat. Genet. 2002, 32, 526-532.

29. Braunschweig, T.; Chung, J.Y.; Hewitt, S.M. Tissue microarrays: Bridging the gap between research and the clinic. Expert Rev. Proteomics 2005, 2, 325-336.

30. Clarke, P.A.; Poele, R.; Wooster, R.; Workman, P. Gene expression microarray analysis in cancer biology, pharmacology, and drug development: Progress and potential. Biochem. Pharmacol. 2001, 62, 1311-1336.

31. Jayapal, M.; Melendez, J.A. DNA microarray technology for target identification and validation. Clin. Exp. Pharmacol. Physiol. 2006, 33, 496-503.

32. Ghallab, Y.H.; Badawy, W. Lab-on-a-Chip: Techniques, Circuits, and Biomedical Applications; Artech House: Norwood, MA, USA, 2010; pp. 1-220. 
33. Kim, J.; Byun, D.; Mauk, M.G.; Bau, H.H. A disposable, self-contained PCR chip. Lab Chip 2009, 9, 606-612.

34. Lab-on-a-Chip: Miniaturized Systems for (Bio)Chemical Analysis and Synthesis, 2nd ed.; Oosterbroek, E., Van den Berg, A., Eds.; Elsevier Science: Amsterdam, The Netherlands, 2003; pp. 1-402.

35. Stybayeva, G.; Mudanyali, O.; Seo, S.; Silangcruz, J.; Macal, M.; Ramanculov, E.; Dandekar, S.; Erlinger, A.; Ozcan, A.; Revzin, A. Lensfree holographic imaging of antibody microarrays for high-throughput detection of Leukocyte numbers and function. Anal. Chem. 2010, 82, 3736-3744.

36. Weissleder, R.; Pittet, M.J. Imaging in the era of molecular oncology. Nature 2008, 452, 580-589.

37. Wang, W.; Eddy, R.; Condeelis, J. The cofilin pathway in breast cancer invasion and metastasis. Nat. Rev. Cancer 2007, 7, 429-440.

38. Weissleder, R. Molecular imaging in cancer. Science 2006, 312, 1168-1171.

39. Jaffer, F.A.; Weissleder, R. Molecular imaging in the clinical arena. JAMA 2005, 293, 855-862.

40. Love, Z.; Wang, F.; Dennis, J.; Awadallah, A.; Salem, N.; Lin, Y.; Weisenberger, A.; Majewski, S.; Gerson, S.; Lee, Z. Imaging of mesenchymal stem cell transplant by bioluminescence and PET. J. Nucl. Med. 2007, 48, 2011-2020.

41. MacLaren, D.C.; Toyokuni, T.; Cherry, S.R.; Barrio, J.R; Phelps, M.E.; Herschman, H.R.; Gambhir, S.S. PET imaging of transgene expression. Biol. Psychiatry 2000, 48, 337-348.

42. Kraitchman, D.L.; Heldman, A.W.; Atalar, E.; Amado, L.C.; Martin, B.J; Pittenger, M.F.; Hare, J.M.; Bulte, J.W. In vivo magnetic resonance imaging of mesenchymal stem cells in myocardial infarction. Circulation 2003, 107, 2290-2293.

43. Long, C.M.; Bulte, J.W. In vivo tracking of cellular therapeutics using magnetic resonance imaging. Expert Opin. Biol. Ther. 2009, 9, 293-306.

44. Kraitchman, D.L.; Bulte, J.W. Imaging of stem cells using MRI. Basic Res. Cardiol. 2008, 103, 105-113.

45. Kalish, H.; Arbab, A.S.; Miller, B.R.; Lewis, B.K.; Zywicke, H.A.; Bulte, J.W.; Bryant, L.H., Jr; Frank, J.A. Combination of transfection agents and magnetic resonance contrast agents for cellular imaging: Relationship between relaxivities, electrostatic forces, and chemical composition. Magn. Reson. Med. 2003, 50, 275-282.

46. Muja, N.; Bulte, J. Magnetic resonance imaging of cells in experimental models. Prog. Nucl. Reson. Spectrosc. 2009, 55, 61-77.

47. Salata, O. Applications of nanoparticles in biology and medicine. J. Nanobiotechnol. 2004, 2, 3-9.

48. Bruchez, M.J.; Moronne, M.; Gin, P.; Weiss, S.; Alivisatos, A.P. Semiconductor nanocrystals as fluorescent biological labels. Science 1998, 281, 2013-2016.

49. Vizirianakis, I.S. Nanomedicine and personalized medicine toward the application of pharmacotyping in clinical practice to improve drug-delivery outcomes. Nanomedicine 2011, 7, $11-17$.

50. Kawasaki, E.S.; Player, A. Nanotechnology, nanomedicine, and the development of new, effective therapies for cancer. Nanomedicine 2005, 1, 101-109.

51. Veiseh, O.; Kievit, F.; Ellenbogen, R.G.; Zhang, M. Cancer Cell Invasion: Treatment and Monitoring Opportunities in Nanomedicine. Adv. Drug. Deliv. Rev. 2011, in press. 
52. Maeda, H.; Wu, J.; Sawa, T.; Matsumura, Y.; Hori, K. Tumor vascular permeability and the EPR effect in macromolecular therapeutics: A review. J. Control. Release 2000, 65, 271-284.

53. Farokhzad, O.C.; Langer, R. Impact of nanotechnology on drug delivery. ACS Nano 2009, 3, 16-20.

54. Danhier, F.; Feron, O.; Préat, V. To exploit the tumor microenvironment: Passive and active tumor targeting of nanocarriers for anti-cancer drug delivery. J. Control. Release 2010, 148, 135-146.

55. Tamilvanan, S. Formulation of multifunctional oil-in-water nanosized emulsions for active and passive targeting of drugs to otherwise inaccessible internal organs of the human body. Int. J. Pharm. 2009, 381, 62-76.

56. Maruyama, K. Intracellular targeting delivery of liposomal drugs to solid tumors based on EPR effects. Adv. Drug. Deliv. Rev. 2011, in press.

57. Arias, J.L.; Gallardo, V.; Ruiz, M.A.; Delgado, A.V. Magnetite/poly(alkylcyanoacrylate) (core/shell) nanoparticles as 5-Fluorouracil delivery systems for active targeting. Eur. J. Pharm. Biopharm. 2008, 69, 54-63.

58. Murphy, C.G.; Modi, S. HER2 breast cancer therapies: A review. Biologics Targets Ther. 2009, 3, 289-301.

59. Cirstoiu-Hapca, A.; Bossy-Nobs, L.; Buchegger, F.; Gurny, R.; Delie, F. Differential tumor cell targeting of anti-HER2 (Herceptin $\left.{ }^{\circledR}\right)$ and anti-CD20 (Mabthera®) coupled nanoparticles. Int. J. Pharm. 2007, 331, 190-196.

60. Li, D.; Yu, H.; Huang, H.; Shen, F.; Wu, X.; Li, J.; Wang, J.; Cao, X.; Wang, Q.; Tang, G. FGF receptor-mediated gene delivery using ligands coupled to polyethylenimine. J. Biomater. Appl. 2007, 22, 163-180.

61. Song, S.; Liu, D.; Peng, J.; Sun, Y.; Li, Z.; Gu, J.; Xu, Y. Peptide ligand-mediated liposome distribution and targeting to EGFR expressing tumor in vivo. Int. J. Pharm. 2008, 363, 155-161.

62. Serda, R.E.; Godin, B.; Blanco, E.; Chiappini, C.; Ferrari, M. Multi-stage delivery nano-particle systems for therapeutic applications. Biochim. Biophys. Acta 2011, 1810, 317-329.

63. Rangel-Yagui, C.O.; Pessoa, A.; Tavares, L.C. Micellar solubilization of drugs. J. Pharm. Pharm. Sci. 2005, 8, 147-163.

64. Sarker, D.K. Engineering of nanoemulsions for drug delivery. Curr. Drug Deliv. 2005, 2, 297-310.

65. Husseini, G.A.; PittMicelles, W.G. Nanoparticles for ultrasonic drug and gene delivery. Adv. Drug. Deliv. Rev. 2008, 60, 1137-1152.

66. Haley, B.; Frenkel, E. Nanoparticles for drug delivery in cancer treatment. Urol Oncol. 2008, 26, 57-64.

67. Hughes, G.A. Nanostructure-mediated drug delivery. Nanomedicine 2005, 1, 22-30.

68. Maruyama, K. Intracellular targeting delivery of liposomal drugs to solid tumors based on EPR effects. Adv. Drug. Deliv. Rev. 2011, 63, 161-169.

69 Obata, Y.; Tajima, S.; Takeoka, S. Evaluation of pH-responsive liposomes containing amino acid-based zwitterionic lipids for improving intracellular drug delivery in vitro and in vivo. J. Control. Release 2010, 142, 267-276.

70. Huang, S. Liposomes in ultrasonic drug and gene delivery. Adv. Drug. Deliv. Rev. 2008, 60, $1167-1176$. 
71. Koide, H.; Asai, T.; Hatanaka, K.; Akai, S.; Ishii, T.; Kenjo, E.; Ishida, T.; Kiwada, H.; Tsukada, H.; Oku, N. T cell-independent B cell response is responsible for ABC phenomenon induced by repeated injection of PEGylated liposomes. Int. J. Pharm. 2010, 392, 218-223.

72. Sakamoto, J.H.; Van de Ven, A.L.; Godin, B.; Blanco, E.; Serda, R.E.; Grattoni, A.; Ziemys, A.; Bouamrani, A.; Hu, T.; Ranganathan, S.I.; De Rosa, E.; Martinez, J.O.; Smid, C.A.; Buchanan, R.M.; Lee, S.; Srinivasan, S.; Landry, M.; Meyn, A.; Tasciotti, E.; Liu, X.; Decuzzi, P.; Ferrari, M. Enabling individualized therapy through nanotechnology. Pharmacol. Res. 2010, 62, 57-89.

73. Shekaran, A.; Garcia, A.J. Nanoscale engineering of extracellular matrix-mimetic bioadhesive surfaces and implants for tissue engineering. Biochim. Biophys. Acta 2011, 1810, 350-360.

74. Barnes, C.P.; Sell, S.A.; Boland, E.D.; Simpson, D.G.; Bowlin, G.L. Nanofiber technology: Designing the next generation of tissue engineering scaffolds. Adv. Drug. Deliv. Rev. 2007, 59, 1413-1433.

75. Atala, A.; Lanza, R.; Nerem, R.; Thomson, J.A. Principles of Regenerative Medicine, 2nd ed.; Academic Press: London, UK, 2011; pp. 733-1105.

76. Prakash, S.; Khan, A.; Paul, A. Nanoscaffold based stem cell regeneration therapy: Recent advancement and future potential. Expert Opin. Biol. Ther. 2010, 10, 1649-1661.

(C) 2011 by the authors; licensee MDPI, Basel, Switzerland. This article is an open access article distributed under the terms and conditions of the Creative Commons Attribution license (http://creativecommons.org/licenses/by/3.0/). 